\title{
Cyclic helix B peptide ameliorates acute myocardial infarction in mice by inhibiting apoptosis and inflammatory responses
}

\author{
Cheng Yang ${ }^{1,2,3}$, Chao Zhang $\mathbb{1}^{1,2}$, Jianguo Jia ${ }^{4,5}$, Lingyan Wang ${ }^{6}$, Weitao Zhang ${ }^{1,2}$, Jiawei Li ${ }^{1,2}$, Ming Xu ${ }^{1,2}$, \\ Ruiming Rong ${ }^{1,2,7}$ and Tongyu Zhu ${ }^{1,2}$
}

\begin{abstract}
Cyclic helix B peptide (CHBP) is a peptide derivant of erythropoietin with powerful tissue-protective efficacies in a variety of organ injuries, but without erythropoietic effect. However, the role of CHBP in acute myocardial infarction (AMI) and related mechanisms are not studied yet. In this study, we found in a murine AMI model that the administration of CHBP could ameliorate cardiac injury, increase the survival rate, inhibit cardiomyocyte apoptosis, improve cardiac function and remodeling, and reduce the expression of inflammatory cytokines in the serum and kidney tissue both at $24 \mathrm{~h}$ and 8 weeks following AMI. This study suggests that CHBP has the potential to be used as an effective drug in the treatment of AMl.
\end{abstract}

\section{Introduction}

It is reported by World Health Organization that 17 million deaths (in 57 million global deaths) are attributed to cardiovascular diseases every year, among which ischemic heart disease and congestive heart failure remain the two leading causes ${ }^{1}$. Heart failure results from the reduction or even blockade of blood supply and apoptosis of cardiomyocytes in infarcted areas following acute myocardial infarction (AMI). Given that the terminally differentiated cardiomyocytes are not able to regenerate, the remaining viable cardiomyocytes in non-infarcted areas have to be forced to share a heavier burden to maintain a sufficient cardiac output, thereby leading to myocardial remodeling. In severe cases, however, the remodeling decompensates and becomes pathogenic, and ultimately leads to heart failure ${ }^{2}$. Thus, novel therapeutic

\footnotetext{
Correspondence: Ruiming Rong (rong.ruiming@zs-hospital.sh.cn) or Tongyu Zhu (tyzhu_dr@163.com) (tyzhu@fudan.edu.cn)

'Department of Urology, Zhongshan Hospital, Fudan University, Shanghai 200032, China

${ }^{2}$ Shanghai Key Laboratory of Organ Transplantation, Shanghai 200032, China Full list of author information is available at the end of the article. These authors contributed equally: Cheng Yang, Chao Zhang Edited by I. Lavrik
}

approaches to ameliorating the development of AMI are critical for the improvement of prognosis in patients.

Since the early 1990s, it has emerged that endogenous erythropoietin (EPO) has cytoprotective effects in a wide variety of tissues, including brain, kidney, and heart ${ }^{3}$. Numerous experimental evidences showed cardioprotective effects of EPO in animal models of AMI. However, these findings are not supported by recent clinical trials designed to investigate the safety and efficacy of EPO in the patients with $\mathrm{AMI}^{4}$. To avoid the erythropoietic side effect of EPO, Brines et al. ${ }^{5}$ firstly reported a nonerythropoietic helix B surface peptide (HBSP) in 2008. In the next period, we and others demonstrated the tissueprotective function of HBSP in a variety of organs, such as kidney $^{6-8}$, heart ${ }^{9,10}$, and brain ${ }^{11}$. However, HBSP demonstrated short half-life in human plasma and liver, and fast degradation in vivo. The instability of HBSP restricts its application in vivo ${ }^{5,12}$. Recently, based on the amino acid sequence of HBSP, we designed and synthesized a novel thioether-cyclized helix B peptide (CHBP) with the increased resistance to proteolytic degradation, improved tissue-protective potency, and decreased administrative frequency and dosage ${ }^{12,13}$. Our previous 
studies demonstrated that CHBP could protect against ischemia-reperfusion induced kidney injury and carbon tetrachloride induced liver injury ${ }^{14-17}$. However, whether CHBP could also function in ameliorating cardiac injury following AMI has not been investigated yet.

Therefore, we designed the present study to determine the role of CHBP in AMI therapy and reveal its cardioprotective mechanisms. We also aim to investigate the changes of morphology and cardiac function, as well as the regulation of apoptosis and inflammation by CHBP treatment.

\section{Results}

CHBP reduced tissue injury, myocardial infarct size, and the expression of CK-MB in the serum

The H\&E-stained myocardial sections showed little apoptotic cells and infiltrated inflammatory cells in CHBP-treated mice following $24 \mathrm{~h}$ ischemia injury. However, severe vacuolation and apoptosis with inflammatory cell infiltration were noted in the control group. Following 8-weeks ischemia injury, myofibrillar degeneration with necrotic damage was observed in the control group, and CHBP ameliorated tissue injury with mild inflammatory cell infiltration (Fig. 1a). We also examined the role of CHBP in the reduction of myocardial infarct size following 8-weeks ischemia injury. As shown in Fig. 1b, ischemia induced a significant myocardial injury as denoted by the infarct size in control mice. In contrast, the infarct size was significantly reduced in CHBP-treated mice, when compared with control mice (Fig. 1b). We, furthermore, examined the level of CK-MB in the serum, and the result showed that CHBP significantly decreased the CK-MB level compared to the control group (Fig. 1c). Survival analysis demonstrated that CHBP significantly increased the survival rate after AMI (Fig. 1d).

\section{CHBP-attenuated cardiomyocyte apoptosis}

Cardiomyocyte apoptosis contributes to myocardial ischemia injury ${ }^{18}$. We examined whether CHBP would attenuate myocardial apoptosis following short-term and long-term ischemia injury. Figure 2a showed that ischemia significantly increased the ISEL-positive apoptotic cells in myocardium in the control group compared with the sham group. However, myocardial apoptotic cells were markedly reduced by CHBP compared with the control mice (Fig. 2b).

\section{CHBP improved left ventricular function and remodeling}

Representative M-mode images and echocardiographic parameters in control and CHBP-treated mice after 8weeks ischemia injury were shown in Fig. 3a. The left ventricular end-diastolic volume was significantly larger in the control group than in the CHBP group (Fig. 3b). The ejection fraction was also remarkably increased in the CHBP group (Fig. 3c). The systolic (Fig. 3d) and diastolic
(Fig. 3e) left ventricular anterior wall were significantly thicker following CHBP treatment, as well as the systolic (Fig. 3f) and diastolic (Fig. 3g) posterior wall thickness.

\section{CHBP modulated the inflammatory responses and chemokines expression}

To evaluate the systemic inflammatory response, we detected pro- and anti-inflammatory cytokines in peripheral blood using Luminex assay. At $24 \mathrm{~h}$ post AMI, proinflammatory cytokines, including interlukin (IL)- $1 \alpha$ (Fig. 4a), IL-1 $\beta$ (Fig. 4b), IL-2 (Fig. 4c), IL-6 (Fig. 4g), IL-12p40 (Fig. 4j), IL-12p70 (Fig. 4k), IL-17 (Fig. 4m), interferon (IFN)- $\gamma$ (Fig. 4q), monocyte chemoattractant protein-1 (MCP-1) (Fig. 4s), macrophage inflammatory protein-1 $\alpha$ (MIP- $\alpha)$ (Fig. 4t), and tumor necrosis factor (TNF)- $\alpha$ (Fig. $4 \mathrm{w}$ ) were significantly decreased by CHBP treatment. In contrast, the anti-inflammatory cytokines IL-4 (Fig. 4e), IL-9 (Fig. 4h), and IL-10 (Fig. 4i) were significantly increased in the CHBP-treated group compared to those in the control group. The chemokines KC/CXCL1 (Fig. 4r) and RANTES/CCL5 (Fig. 4v) were significantly increased by CHBP. Eight weeks post AMI, the change of inflammatory cytokines was similar to that at $24 \mathrm{~h}$. IL- $1 \alpha$ (Fig. 5a), IL-1 $\beta$ (Fig. 5b), IL-2 (Fig. 5c), IL-6 (Fig. 5g), IL-12p40 (Fig. 5j), IL12p70 (Fig. 5k), IL-17 (Fig. 5m), IFN- $\gamma$ (Fig. 5q), MCP-1 (Fig. 5s), MIP- $\beta$ (Fig. 5u), and TNF- $\alpha$ (Fig. $5 w$ ) were significantly decreased by CHBP treatment, whereas IL-4 (Fig. 5e), IL-9 (Fig. 5h), and IL-10 (Fig. 5i) were remarkably by CHBP treatment. Unlike to the $24 \mathrm{~h}$ post AMI, the $\mathrm{KC} /$ CXCL1 expression was significantly increased in the CHBP group (Fig. 5r).

To evaluate the local inflammation responses, we also examined the typical inflammatory cytokines mRNA expression in the myocardium by using RT-qPCR. The mRNA expression of TNF- $\alpha$ (Fig. 6a) and IFN- $\gamma$ (Fig. 6c) was significantly decreased by CHBP treatment at both $24 \mathrm{~h}$ and 8 weeks. IL-1 $\beta$ (Fig. 6b) and IL-12p40 (Fig. 6d) mRNA level was significantly reduced in the myocardium. The mRNA expression of IL-4 (Fig. 6e) and IL-10 (Fig. 6f) was significantly increased at $24 \mathrm{~h}$ and 8 weeks in the CHBP-treated group, respectively.

\section{Discussion}

This study demonstrated that the novel peptide CHBP exerted cardioprotective effect in a murine AMI model. CHBP significantly ameliorated histological injury and left ventricular remodeling. CHBP also inhibited cardiomyocytes apoptosis and inflammation.

CHBP is a thioether-cyclized peptide with conformational constraining and firstly reported by our group in a kidney ischemia-reperfusion mode ${ }^{12}$. Although its parent protein erythropoietin (EPO) has been demonstrated to alleviate heart injury and promote heart repair in the animal studies about $\mathrm{AMI}^{19,20}$, the large REVEAL study showed no 


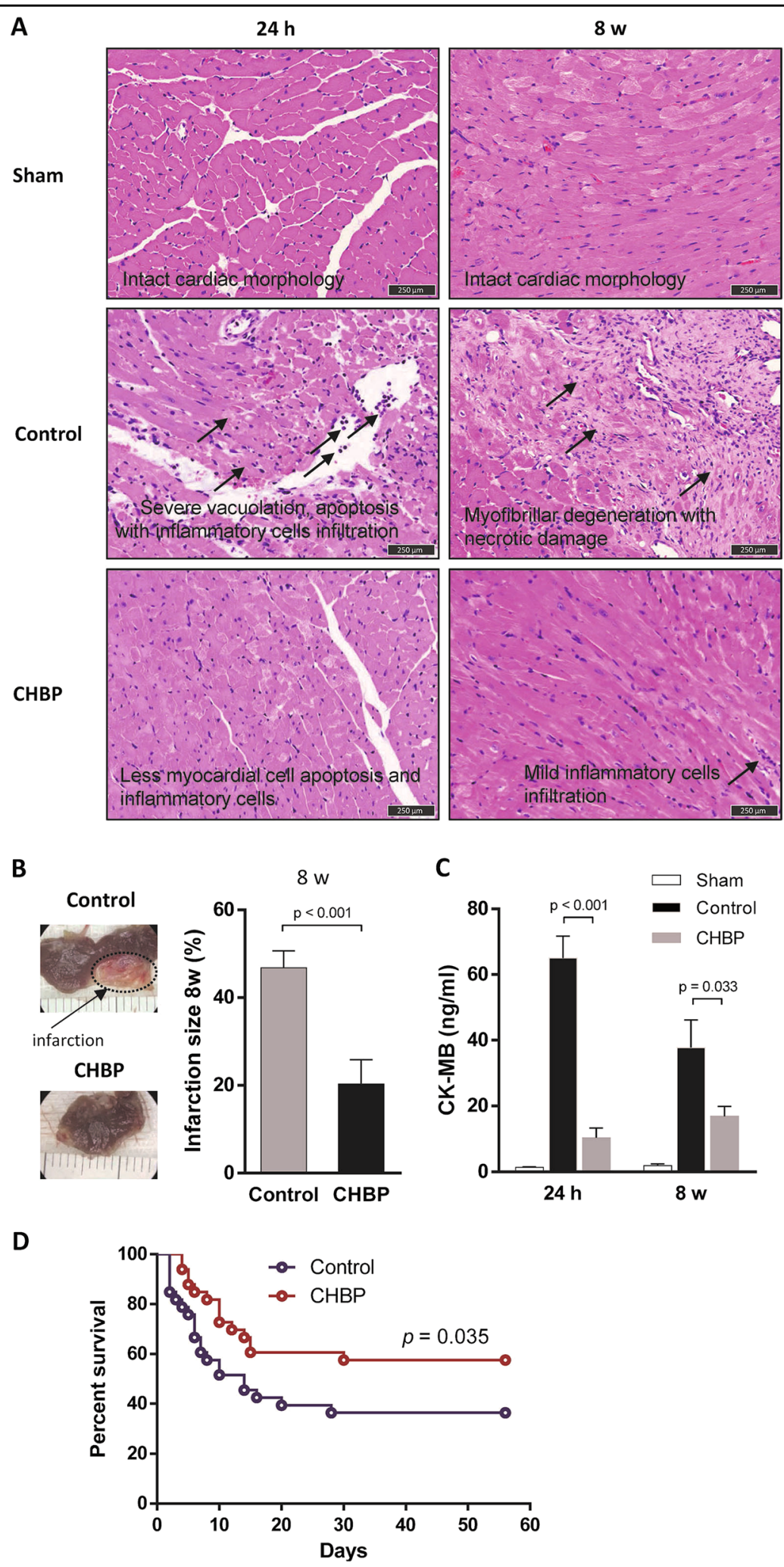

Fig. 1 CHBP ameliorated cardiac injury. The H\&E staining showed CHBP significantly attenuated cardiac injury in terms of less vacuolation, myofibrillar degeneration, apoptosis and necrosis, as well as less inflammatory cells infiltration post $24 \mathrm{~h}$ and 8-weeks AMI (a). Ischemia induced significant myocardial injury as denoted by the infarct size in control mice. In contrast, infarct size was significantly reduced in CHBP-treated mice, when compared with control mice (b). The expression of CK-MB in the serum was also significantly decreased by CHBP treatment (c). In addition, CHBP also remarkably increased the survival rate after AMI (d) 


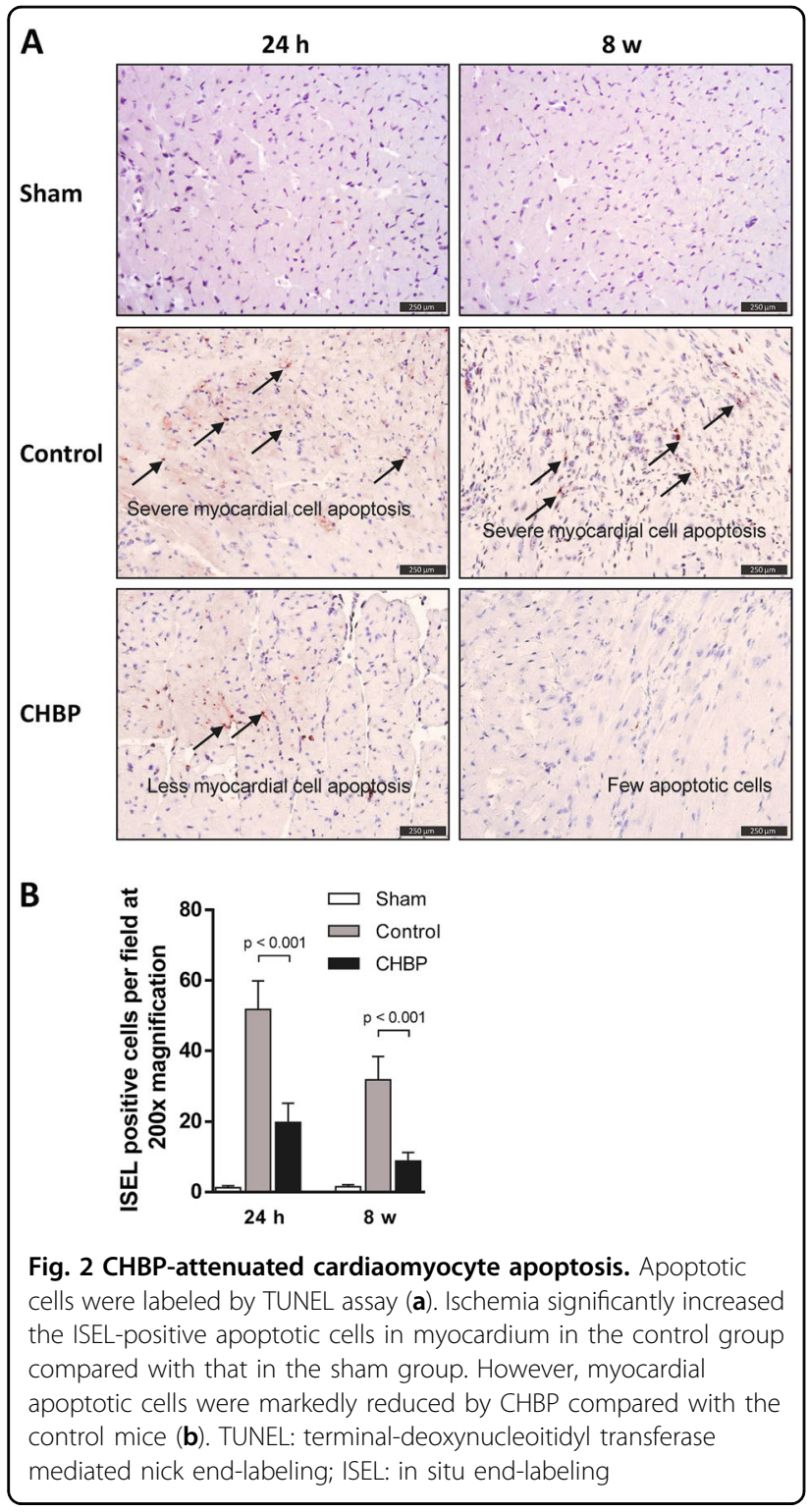

reduction of infarct size following EPO treatment ${ }^{21}$. Recently, a meta-analysis indicated that short-term administration of EPO in the patients with myocardial infarction did not result in an improved cardiac function, reduced infarct size and lower all-cause mortality ${ }^{22}$. Similarly, the results of a randomized clinical trial indicated that low-dose EPO also did not improve cardiac function in the patients with ST-segment elevation myocardial infarction ${ }^{23}$. Lowdose EPO therapy may not be the choice of treatment for the patients with AMI, while a higher dose of EPO might be more effective ${ }^{24}$. However, high-dose EPO therapy would take higher risks of side effects, such as erythrocytosis, thrombogenesis and hypertension ${ }^{25,26}$. To avoid the abovementioned side effects while maintain the tissueprotective efficacy of EPO, Brines et al. firstly reported a linear peptide helix B surface peptide (HBSP), which entirely replicated the cardioprotective properties from the parent erythropoietin, but without erythropoiesis ${ }^{9,10}$. Unfortunately, HBSP was proved to be very unstable both in vivo and in vitro. Owing to its short plasma half-life (about $9 \mathrm{~min}$ ), linear HBSP needs to be frequently administered with high doses to achieve tissue-protective effects, and that would restrict its clinical application ${ }^{12}$. Therefore, we designed and synthesized CHBP with better stability and stronger tissue-protective property ${ }^{16,27-30}$. This study confirmed the cardioprotective effect of CHBP.

There are some limitations in the present study. For instance, the molecular mechanism involved in the regulation of apoptosis and inflammation by CHBP needs to be further investigated. To evaluate the long-term cardioprotective effects of CHBP from bench to bedside, more samples and longer observation are still required.

In conclusion, our study reveals that the novel peptide CHBP protects heart against AMI in terms of better heart function, less cardiomyocytes injury and apoptosis, and low level of inflammation.

\section{Materials and methods \\ Animals and surgery}

Male C57B6 mice (weighing 20-25 g) were obtained from Shanghai Slac Lab Animal, Co., Ltd., and bred in an experimental animal room of SPF grade. Mice were anaesthetized ( $2 \%$ isoflurane), intubated and ventilated, and then were placed on an adjustable heating pad to maintain a core temperature of $36-37^{\circ} \mathrm{C}$. The heart was exposed through the fourth intercostal space separated with an adjustable microretractor (Medicon eG, Tuttlingen, Germany) and the left anterior descendent coronary artery was (LAD) ligated using 10/0 silk suture (Ethicon Endosurgery, OH, USA), $1 \mathrm{~mm}$ distal to left atrial appendage. Successful performance of coronary occlusion was verified by the visible pale of afflicted tissue and QRS alterations within the first seconds of occlusion. The sham-operated animals underwent the same surgical procedures except that the suture passing the LAD was not fastened. After surgery, buprenorphine $(0.05 \mathrm{mg} / \mathrm{kg}$ per $6 \mathrm{~h}$, subcutaneously) was given for $48 \mathrm{~h}$. Immediately after surgery, the mice were injected with $30 \mu \mathrm{g} / \mathrm{kg}$ CHBP i.p. for a single dose. For short-term therapeutic effect evaluation, mice were randomly divided into three groups $(n=10)$ : (1) sham group; (2) control group; (3) CHBP group: with one dose of CHBP administration. All mice were sacrificed at $24 \mathrm{~h}$ post surgery. For long-term therapeutic effect evaluation, mice were also randomly divided into three groups $(n=33)$ : (1) sham group; (2) control group; (3) CHBP group: with $30 \mu \mathrm{g} /$ $\mathrm{kg}$ CHBP administration three times a week. All mice were sacrificed at 8-weeks post surgery. All animal procedures were performed according to the guidelines of the Care and Use of the Laboratory Animal Ethical Commission of Fudan University. 

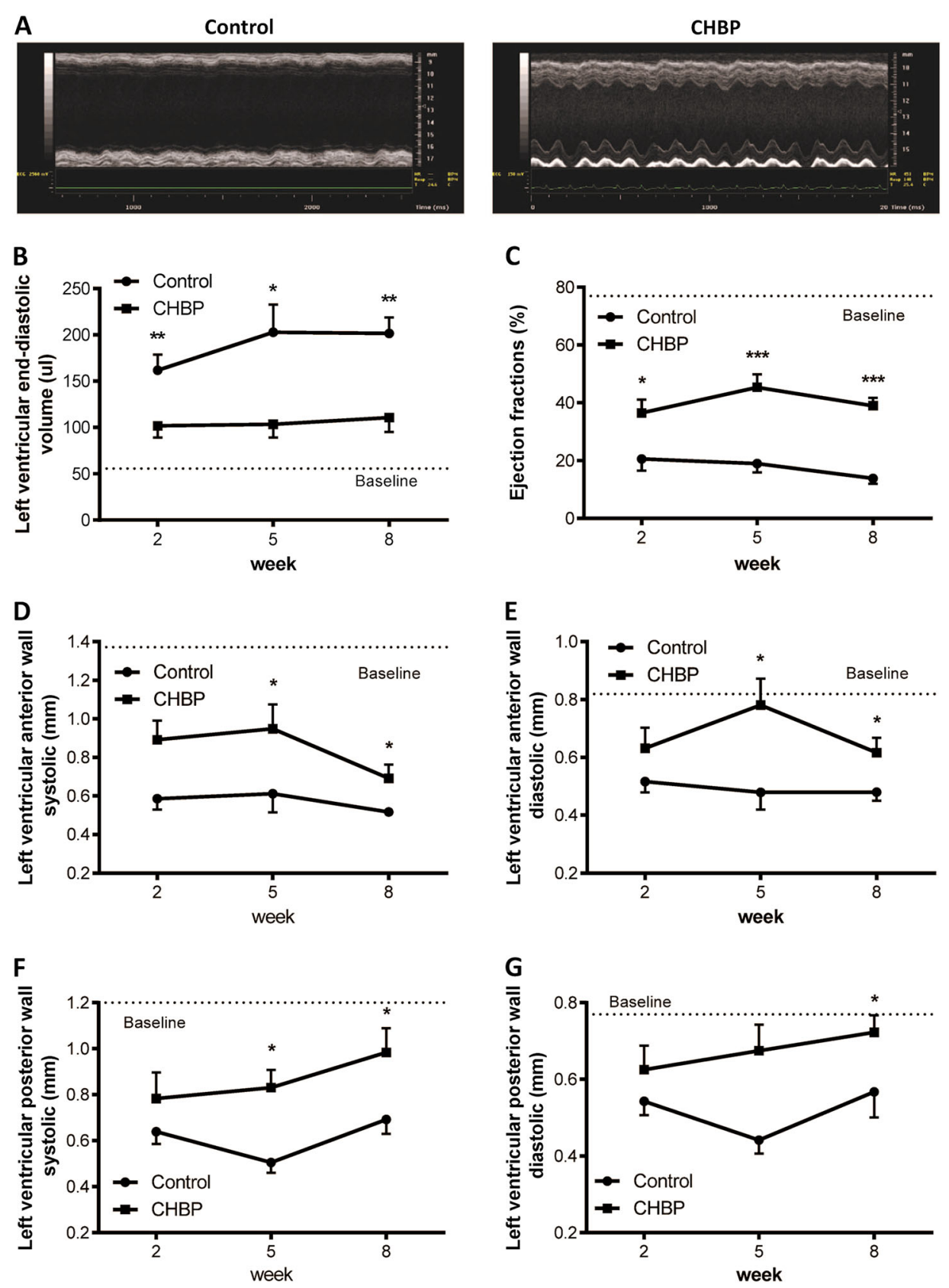

Fig. 3 CHBP improved left ventricular function and remodeling. Representative M-mode images and echocardiographic parameters in control and CHBP-treated mice after 8-weeks ischemia injury are shown (a). The left ventricular end-diastolic volume was significantly larger in the control group compared to that in the CHBP group (b). The ejection fraction was also remarkably improved in the CHBP group (c). The systolic (d) and diastolic (e) left ventricular anterior wall were significantly thicker by CHBP treatment, as well as the systolic (f) and diastolic (g) posterior wall thickness. The dot line represents baseline parameters, which were measured by sham mice

\section{Histology}

Hearts were arrested with $15 \% \mathrm{KCl}$ and sectioned into 3-4 transverse slices, cut parallel to the atrioventricular ring. Each slice was fixed with $4 \%(\mathrm{v} / \mathrm{v})$ buffered formalin, embedded in paraffin, and sectioned into $5 \mu \mathrm{m}$ sections. Serial sections were stained with hematoxylin and eosin (H\&E; Sigma).

\section{Enzyme-linked immunosorbent assay (ELISA) assay}

Creatine kinase-MB (CK-MB) was assayed in duplicate using sandwich ELISA (Quantikine Kit for Mice CK-MB Immunoassay; Uscn Life Science Inc., Houston, TX, USA). The sample preparation and procedure were performed according to the manufacturer's instructions. 

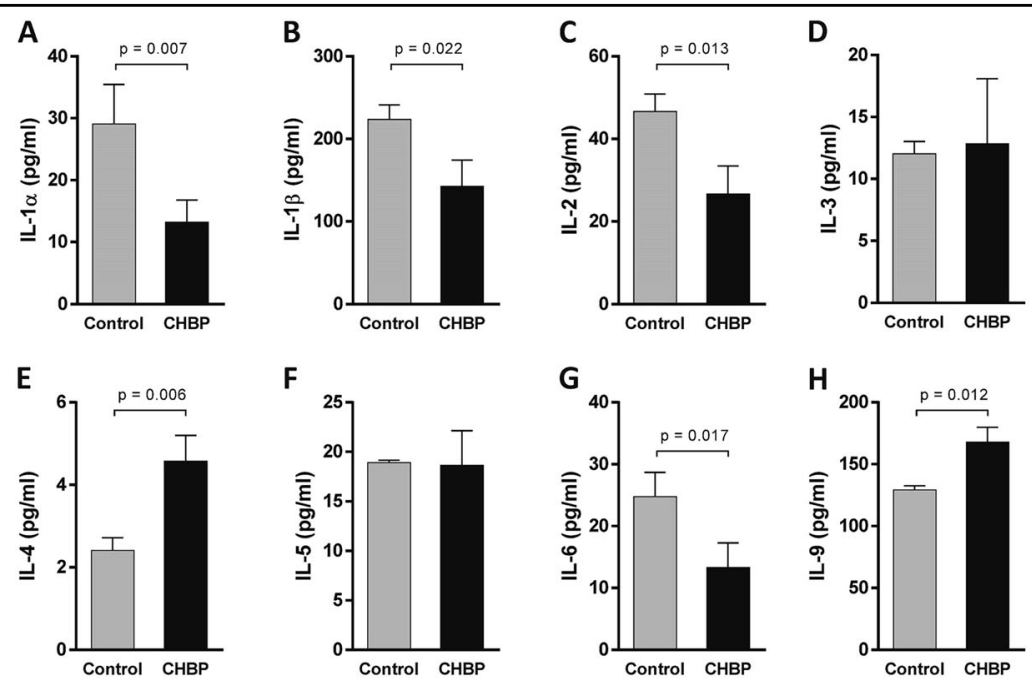

$\mathbf{F}$

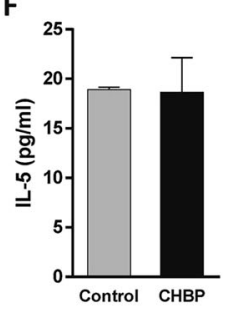

G
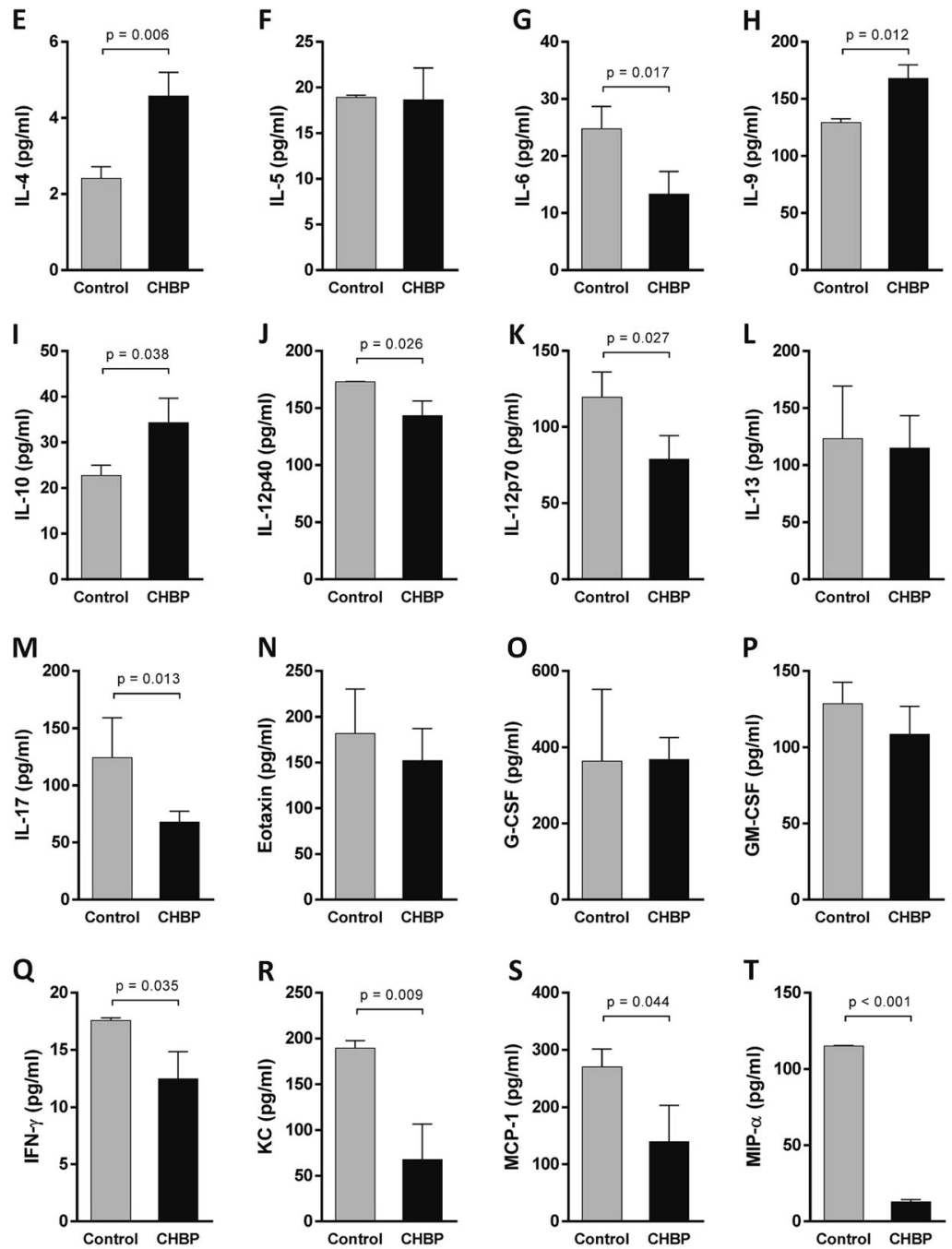

T
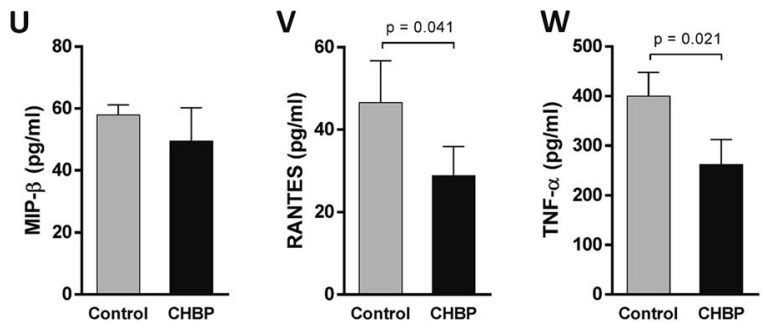

Fig. 4 The inflammatory cytokines and chemokines expression at $\mathbf{2 4} \mathbf{h}$ post AMI. Pro-inflammatory cytokines, including IL-1a (a), IL-1 3 (b), IL-2

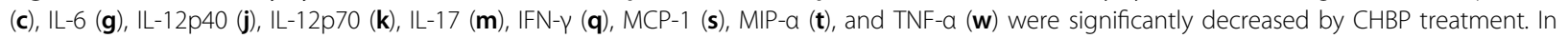
contrast, the anti-inflammatory cytokines IL-4 (e), IL-9 (h), and IL-10 (i) were significantly increased in the CHBP-treated group compared to those in the control group. The chemokines KC/CXCL1 ( $\mathbf{r}$ ) and RANTES/CCL5 (v) were significantly increased by CHBP. The expressions of IL-3 (d), IL-5 (f), IL-13 (I), Eotaxin (n), G-CSF (o), GM-CSF (p) and MIP- $\beta$ (u) were not significantly different between the control and CHBP group 

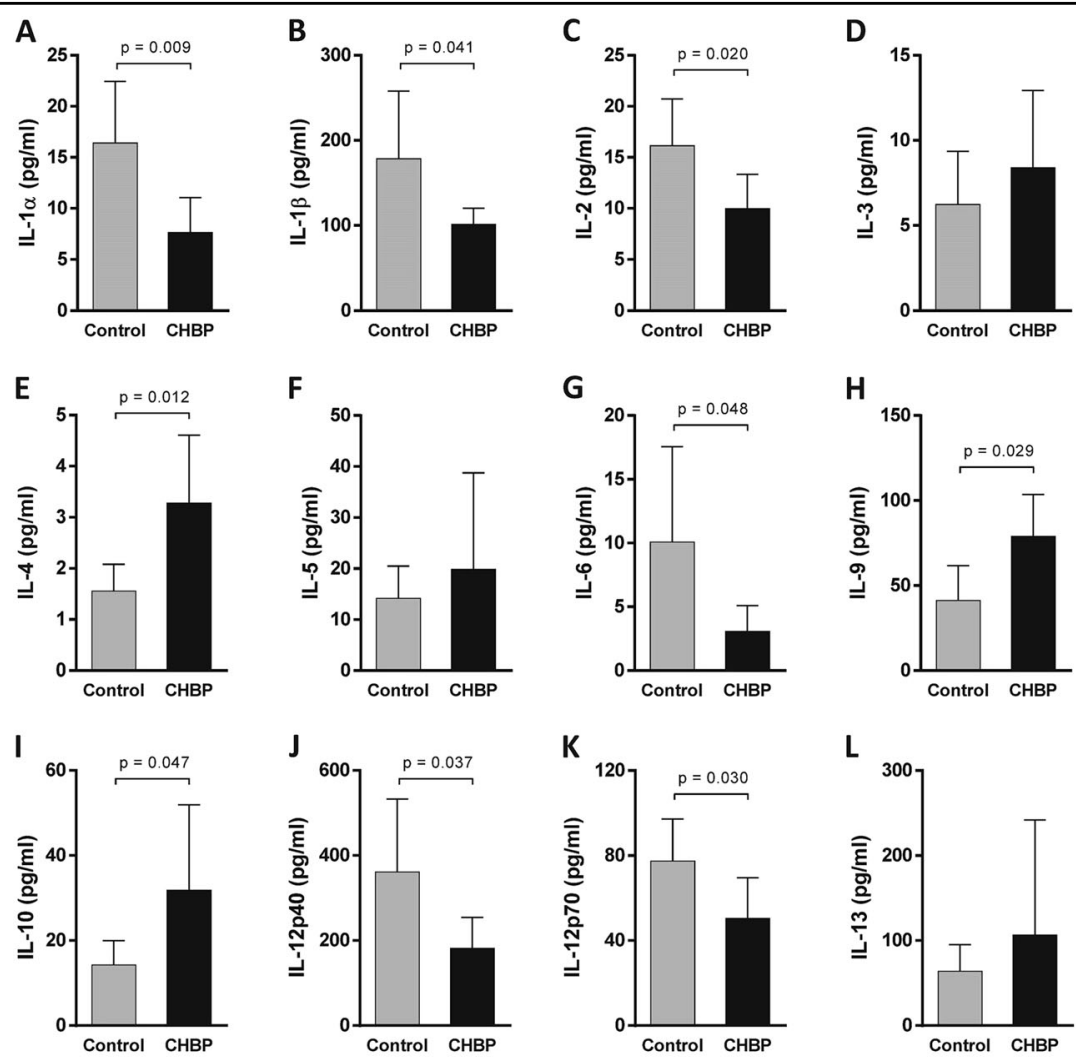

K

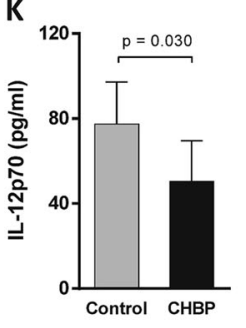

L
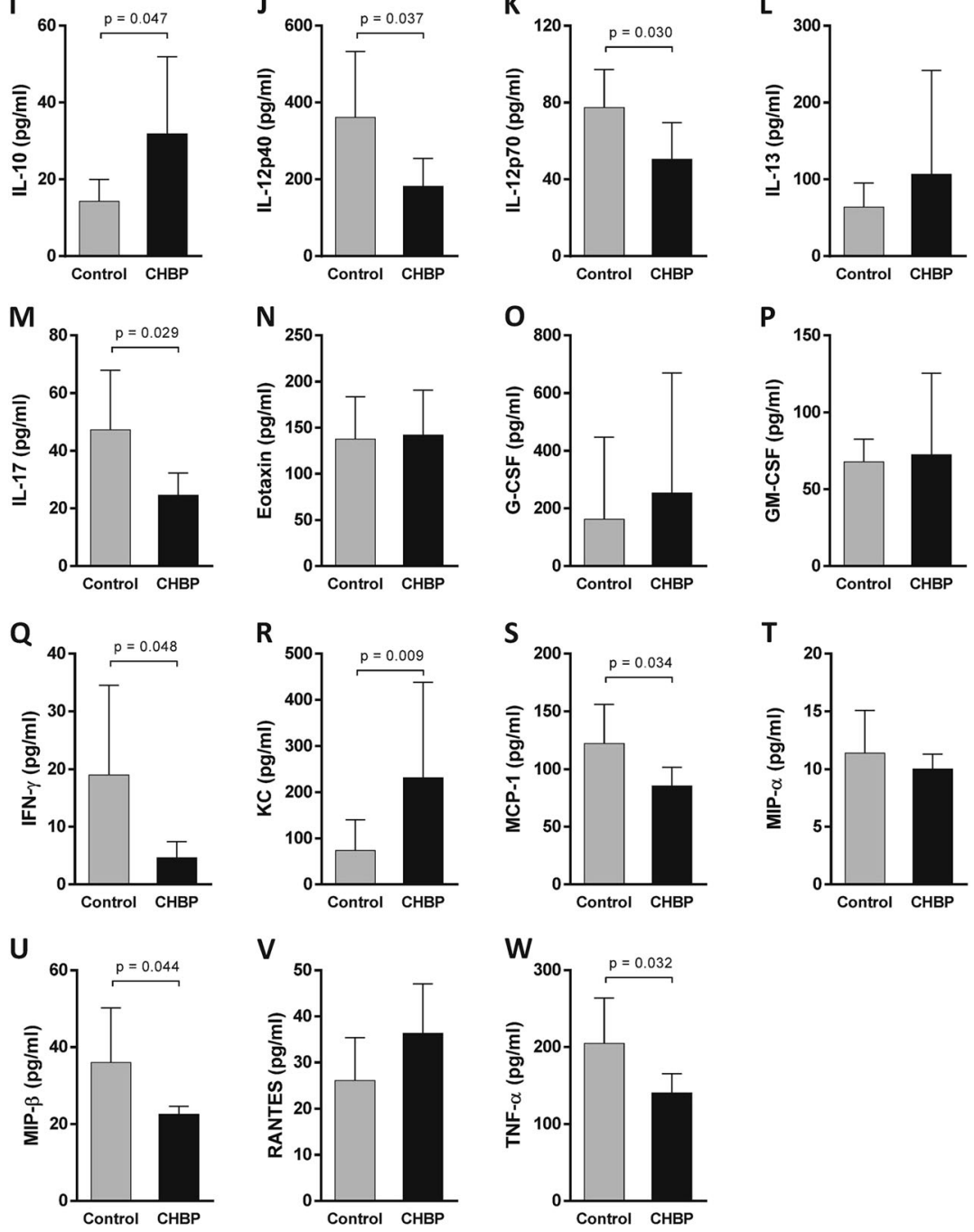

Fig. 5 The inflammatory cytokines and chemokines expression at 8-weeks post AMI. The expression of IL-1a (a), IL-1 3 (b), IL-2 (c), IL-6 (g), IL-

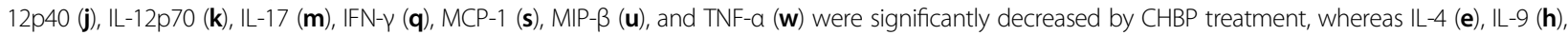
and IL-10 (i) were remarkably by CHBP treatment. The KC/CXCL1 (r) expression was significantly increased in the CHBP group. The expressions of IL-3 (d), IL-5 (f), IL-13 (I), Eotaxin (n), G-CSF (o), GM-CSF (p), MIP-a (t) and RANTES (v) were not significantly different between the control and CHBP group 

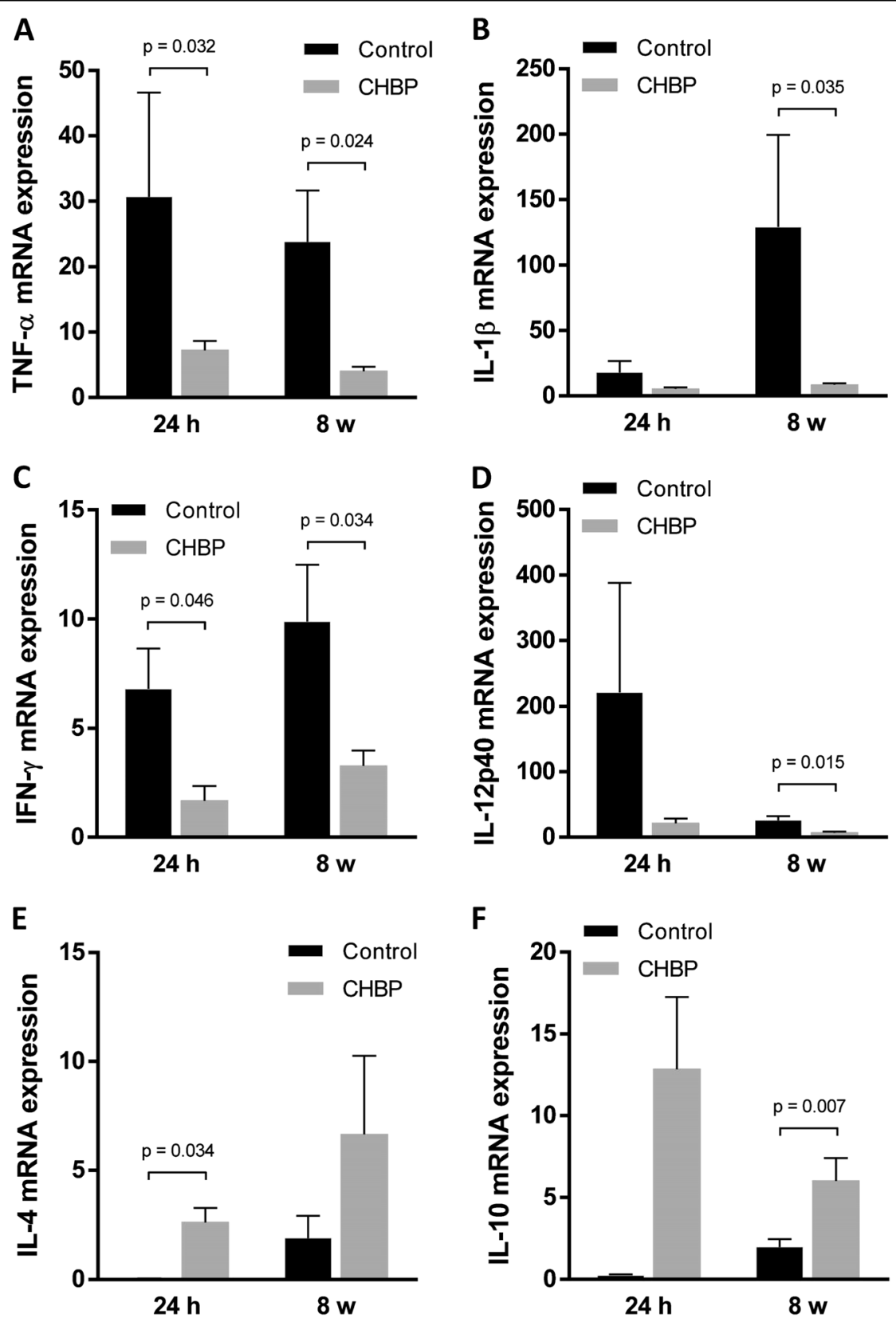

Fig. 6 The inflammatory cytokines expression at mRNA level post AMI. The mRNA expression of TNF-a (a) and IFN- $\gamma$ (c) was significantly decreased by CHBP treatment at both $24 \mathrm{~h}$ and 8 weeks. IL-1 $\beta$ (b) and IL-12p40 (d) mRNA level was significantly reduced in myocardium. The mRNA expression of IL-4 (e) and IL-10 (f) was significantly increased at $24 \mathrm{~h}$ and 8 weeks in the CHBP-treated group, respectively

In situ end-labeling (ISEL) apoptotic cells

ISEL apoptotic cells were detected using a TUNEL Apoptosis Detection Kit (Millipore, MA, USA). Paraffin sections of $4 \mu \mathrm{m}$ were digested by $40 \mu \mathrm{g} / \mathrm{mL}$ of proteinase K (EMD Chemicals, NJ, USA) for $15 \mathrm{~min}$ at $37^{\circ} \mathrm{C}$, incubated with TdT and digoxigenin-dUTP at $37^{\circ} \mathrm{C}$ for 60 min, and transferred to a wash/stop buffer for $30 \mathrm{~min}$. After adding anti-digoxigenin-peroxidase complex for 30 min, the tissue sections immersed in buffer were developed by 3'-amino-9-ethylcarbazole (AEC, DAKO, Carpinteria, USA) substrate (dark red color). Apoptotic cells were examined at $\times 400$ magnification in 20 fields for semi-quantitation.

\section{Transthoracic echocardiographic analysis}

Echocardiographic measurements were performed with a Vevo 770 system (Visual Sonics, Toronto, Ontario, Canada) applied to the shaved chest wall of mice 
anesthetized with isoflurane. All measurements, averaged for three consecutive cardiac cycles, were performed by an experienced technician and reviewed by a cardiologist; all were blinded to the treatment groups. The ejection fraction (EF), left ventricular end-diastolic volume, left ventricular systolic and diastolic anterior, and posterior wall thickness were measured in M-mode recordings.

\section{Cytokine multiplex analysis}

Serum samples were analyzed for 23 cytokines using a Bio-Plex Multiplex System (Bio-Rad, Hercules, CA, USA) according to the manufacturer's protocol.

\section{Real-time quantitative polymerase chain reaction (RT- qPCR)}

Total RNA was extracted from mice kidneys with Trizol reagent (Invitrogen, Carlsbad, USA) according to the manufacturer's instructions. Total RNA ( 3 to $5 \mu \mathrm{g}$ ) was transcribed into complementary DNA by the Superscript II reverse transcriptase (Invitrogen) and random primer oligonucleotides (Invitrogen). Gene-specific primers for mice, such as TNF- $\alpha$, IL- $1 \beta$, IFN- $\gamma$, IL-12p40, IL-4, and IL-10 and GAPDH, were designed based on the sequences available from PubMed (Table S1 contains the list of sequences). RT-qPCR was performed in a MasterCycler RealPlex4 system (Eppendorf, Hamburg, Germany) in combination with the Absolute QPCR SYBR Green premix (Takara Bio Inc., Tokyo, Japan). After a hot start (30 s at $95^{\circ} \mathrm{C}$ ), the amplification parameters were as follows: $5 \mathrm{~s}$ at $95^{\circ} \mathrm{C}, 30 \mathrm{~s}$ at $55^{\circ} \mathrm{C}$, and $60 \mathrm{~s}$ at $72^{\circ} \mathrm{C}$ for 45 cycles. The expression levels, which were normalized with GAPDH, were calculated according to the housekeeping gene GAPDH using the $2^{-\Delta \Delta \mathrm{Ct}}$ method.

\section{Statistical analysis}

Data are presented as mean \pm standard deviation (SD). Statistical analysis (SPSS 18.0 software, SPSS Inc, Armonk, NY, USA) was performed with the two-tailed independent Student's $t$-test after the demonstration of homogeneity of variance with the $F$-test or one-way ANOVA for more than two groups. The Scheffe test was used for post-hoc analysis. Statistical significance was set as $p<0.05$.

\section{Acknowledgements \\ This study was supported by National Natural Science Foundation of China (81770746 to Cheng Yang, 81770747 to Ruiming Rong and 81873621 to Tongyu Zhu), National Key R\&D Program of China (2018YFA0107501 to Ruiming Rong, 2018 YFA0107502 to Cheng Yang), Shanghai Rising-Star Program (19QA1406300 to Cheng Yang) and Medical and Health Talents Training Plan for the Excellent Youth of Shanghai Municipal (2018YQ50 to Cheng Yang).}

\section{Author details}

'Department of Urology, Zhongshan Hospital, Fudan University, Shanghai 200032, China. ${ }^{2}$ Shanghai Key Laboratory of Organ Transplantation, Shanghai 200032, China. ${ }^{3}$ Zhangjiang Institute of Fudan University, Shanghai 201203,
China. ${ }^{4}$ Department of Cardiology, Zhongshan Hospital, Fudan University, Shanghai 200032, China. ${ }^{5}$ Shanghai Institute of Cardiovascular Diseases, Shanghai 200032, China. ${ }^{6}$ Biomedical Research Center, Zhongshan Hospital, Fudan University, Shanghai 200032, China. 'Department of Transfusion, Zhongshan Hospital, Fudan University, Shanghai 200032, China

\section{Author's contributions}

Y.C. conceived of the study, and participated in the design of the study and performed the statistical analysis. Z.C. and J.J. carried out the animal surgery. $Y$. C., L.J., Z.W., and W.L. performed the pathological and molecular studies. Y.C. drafted the manuscript. Z.T. participated in the design of the study and revised the manuscript. Y.C., R.R., and Z.T. provided the fund support. All authors have read and approved the final manuscript.

\section{Conflict of interest}

The authors declare that they have no conflict of interest.

\section{Publisher's note}

Springer Nature remains neutral with regard to jurisdictional claims in published maps and institutional affiliations.

Received: 9 February 2019 Revised: 22 February 2019 Accepted: 4 March 2019

Published online: 18 March 2019

\section{References}

1. Musa, S., Xin, L. Z., Govindasamy, V., Fuen, F. W. \& Kasim, N. H. Global search for right cell type as a treatment modality for cardiovascular disease. Expert. Opin. Biol. Ther. 14, 63-73 (2014).

2. Perricone, A. J. \& Vander Heide, R. S. Novel therapeutic strategies for ischemic heart disease. Pharmacol. Res. 89C, 36-45 (2014).

3. Brines, M. \& Cerami, A. Erythropoietin-mediated tissue protection: reducing collateral damage from the primary injury response. J. Intern. Med. 264, 405-432 (2008).

4. Roubille, F. et al. What is the role of erythropoietin in acute myocardial infarct? Bridging the gap between experimental models and clinical trials. Cardiovasc. Drugs Ther. 27, 315-331 (2013).

5. Brines, M. et al. Nonerythropoietic, tissue-protective peptides derived from the tertiary structure of erythropoietin. Proc. Natl Acad. Sci. USA 105, 10925-10930 (2008).

6. Yang, C. et al. Helix B surface peptide administered after insult of ischemia reperfusion improved renal function, structure and apoptosis through beta common receptor/erythropoietin receptor and PI3K/Akt pathway in a murine model. Exp. Biol. Med. 238, 111-119 (2013).

7. Wu, Y. et al. Protective effects of HBSP on ischemia reperfusion and cyclosporine a induced renal injury. Clin. Dev. Immunol. 2013, 758159 (2013).

8. Patel, N. S. et al. Delayed administration of pyroglutamate helix B surface peptide (pHBSP), a novel nonerythropoietic analog of erythropoietin, attenuates acute kidney injury. Mol. Med. 18, 719-727 (2012).

9. Ahmet, I. et al. A small nonerythropoietic helix B surface peptide based upon erythropoietin structure is cardioprotective against ischemic myocardial damage. Mol. Med. 17, 194-200 (2011).

10. Ueba, $\mathrm{H}$. et al. Cardioprotection by a nonerythropoietic, tissue-protective peptide mimicking the 3D structure of erythropoietin. Proc. Natl Acad. Sci. U.S. A. 107, 14357-14362 (2010).

11. Patel, N. S. A. et al. A nonerythropoietic peptide that mimics the $3 \mathrm{D}$ structure of erythropoietin reduces organ injury/dysfunction and inflammation in experimental hemorrhagic shock. Mol. Med. 17, 883-892 (2011).

12. Yang, C. et al. A novel proteolysis-resistant cyclic helix B peptide ameliorates kidney ischemia reperfusion injury. Biochim. Biophys. Acta 1842, 2306-2317 (2014).

13. Yang, C. et al. Cyclic helix B peptide in preservation solution and autologous blood perfusate ameliorates ischemia-reperfusion injury in isolated porcine kidneys. Transplant. Direct 1, 1-9 (2015).

14. Yang, C. et al. Proteome analysis of renoprotection mediated by a novel cyclic helix B peptide in acute kidney injury. Sci. Rep. 5, 18045 (2015). 
15. Yang, $C$. et al. Cyclic helix B peptide inhibits ischemia reperfusioninduced renal fibrosis via the PI3K/Akt/FoxO3a pathway. J. Transl. Med. 13, 355 (2015)

16. Zhang, C., Yang, C. \& Zhu, T. From erythropoietin to its peptide derivatives: smaller but stronger. Curr. Protein Pept. Sci. 18, 1191-1194 (2017).

17. Wu, S. et al. The protective effects of helix B surface peptide on experimental acute liver injury induced by carbon tetrachloride. Dig. Dis. Sci. 62, 1537-1549 (2017).

18. van Empel, V. P. et al. Myocyte apoptosis in heart failure. Cardiovasc. Res. 67, 21-29 (2005).

19. Sanchis-Gomar, F. et al. Erythropoietin and the heart: physiological effects and the therapeutic perspective. Int. J. Cardiol. 171, 116-125 (2014).

20. Zafiriou, M. P. et al. Erythropoietin responsive cardiomyogenic cells contribute to heart repair post myocardial infarction. Stem Cells $\mathbf{3 2}$, 2480-2491 (2014).

21. Najjar, S. S. et al. Intravenous erythropoietin in patients with ST-segment elevation myocardial infarction: REVEAL: a randomized controlled trial. JAMA 305, 1863-1872 (2011)

22. Yang, H. T., Xiu, W. J., Zheng, Y. Y., Ma, Y. T. \& Xie, X. Effects of erythropoietin on the clinical outcomes of patients with acute ST segment elevation myocardial infarction after percutaneous coronary intervention: A meta-analysis. Int. J. Clin. Pharmacol. Ther. 56, 277-279 (2018).
23. Minamino, T. et al. Low-dose erythropoietin in patients with st-segment elevation myocardial infarction (EPO-AMI-II)- a randomized controlled clinical trial. Circ. J. 82, 1083-1091 (2018).

24. Ali-Hassan-Sayegh, S. et al. Administration of erythropoietin in patients with myocardial infarction: does it make sense? An updated and comprehensive meta-analysis and systematic review. Cardiovasc. Revasc. Med. 16, 179-189 (2015).

25. Lippi, G., Franchini, M. \& Favaloro, E. J. Thrombotic complications of erythropoiesis-stimulating agents. Semin. Thromb. Hemost. 36, 537-549 (2010).

26. Krapf, R. \& Hulter, H. N. Arterial hypertension induced by erythropoietin and erythropoiesis-stimulating agents (ESA). Clin. J. Am. Soc. Nephrol.: CJASN 4, 470-480 (2009).

27. Liu, A. et al. TRPM7 in CHBP-induced renoprotection upon ischemia reperfusion-related injury. Sci. Rep. 8, 5510 (2018).

28. Zeng, $Y$. et al. Protective effects of cyclic helix $B$ peptide on aristolochic acid induced acute kidney injury. Biomed. Pharmacother. 94, 1167-1175 (2017).

29. Wang, S. et al. A novel cytoprotective peptide protects mesenchymal stem cells against mitochondrial dysfunction and apoptosis induced by starvation via Nrf2/Sirt3/FoxO3a pathway. J. Transl. Med. 15, 33 (2017).

30. Yang, C. et al. A novel cyclic helix B peptide inhibits dendritic cell maturation during amelioration of acute kidney graft rejection through Jak-2/STAT3/ SOCS1. Cell Death Dis. 6, e1993 (2015). 